\title{
sur modèle réduit du port de Port-en-Bessin
}

\section{Study of Port-en-Bessin harbour on a scale model}

\author{
PAR C. BIRARD
}

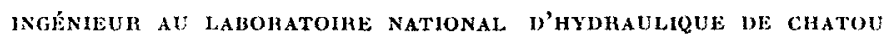

L'étude sur modèle réduit du port de Port-enBessin a permis de proposer des ouvrages"d'un type nouveau pour diminuer l'agitation dans le port.

Ces ouvrages appeless «Résonateurs》 et mis au point au Laboratoire National d'Hydraulique de Chatou, utilisent le caractère ondulatoire de la houle pour la réfléchir suns obstacle frontal.

De construction très simple et peu onéreuse, ils donnent d'excellents résultats là où souvent les solutions classiques sont impuissantes. La seule condition qui limite letur emploi est la largeur de In persse à protéger. Elle ne doit pas ètre trop grande par rapporl à la longueur d'onde de la houle.

\section{I. - Introduction}

A.

Port-en-Bessin (fig. 1), situé dans le Calvados à l'ouest de l'embouchure de l'Orne, est un port de pêche assez important. Etabli sur une côte rectiligne, il semble à première vue assez bien protégé par deux importantes jetées à l'est et à l'ouest. Malheureusement, dès que la mer devient mauvaise, la houle pénètre par la passe d'entrée, se réfléchit sur les quais et les jetées à parois verticales pour entretenir une agitation désordonnce du plan d'eau. Les Instructions Nautiques de la Marine recommandent d'ailleurs de ne point y faire relâche Iors des tempètes.

Pourtant, deux bassins successifs communiquant avec l'avant-port par un chenal de $100 \mathrm{~m}$ de long et $10 \mathrm{~m}$ de large paraissent constituer un abri sûr. En fait, la houle pénètre dans le convergent précédant le chenal, se cambre fortement et
The study of Port-en-bessin harbour on a scale model has allowed a new type of structure to be proposed for accrasing the agitation in the port.

These structures called "résonateurs", whirh were developed by the Laboratoire National d'Hydraulique at Chatou, use the character of the wave motion to reflect the wave, without any frontal obstacle.

Their construction is very simple and inexpensive, and they give excellent results, often where the classical solutions are of no nse. The only condition limiting their use is the widlh of the pass to be protected. The pass must not be too large relation to the lenglh of the incident wave. contraint les bateaux à s'entasser dans le second bassin où ils ne trouvent cependant qu'un calme tout relatif.

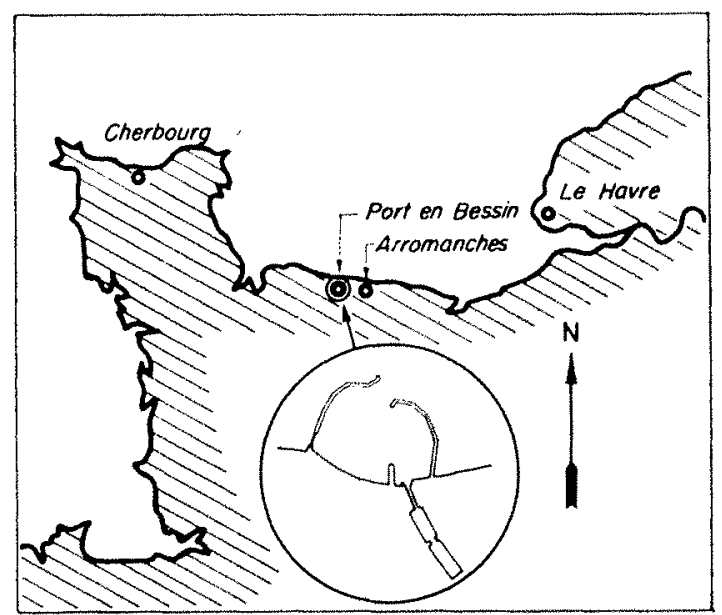

Fra. 1. ... Situation de Port-en-Bessin. 
D'autre part, le développement du port conduit à rechercher une augmentation de la longueur de quais utilisables en tout temps, en particulier on nous a demandé d'assurer la protection de l'Epi de la Poissonnerie qui, établi en eau relativement profonde, permettrait d'accélérer les opérations de déchargement du poisson.

En définitive, les problèmes posés étaient:

1. Rechercher des ouvrages protégeant très efficacement les bassins;

2. Examiner les possibilités d'amélioration du plan d'eau dans la partie de l'avant-port comprise entre la jetée de l'Est et l'Epi de la Poissonnerie.

\section{B. - LES DONNÉES DU PROBLEME.}

L'étude sur modèle réduit de ce port, faite ḋ la demande de l'Administration des Ponts et Chaussées du Calvados, a été précédée par un certain nombre de mesures sur place qui ont permis de préciser les données du problème : périodes ( 5 à $9 \mathrm{~s}$ ) et direction des houles dangereuses (N,-O. à N.-E.)

\section{II. - Modèle réduit}

\section{A. - Chorx de L'ÉChelle.}

Les renseignements recueillis dans la nature permettent de fixer l'échelle minima à utiliser pour la construction du modèle. Il faut en effet, pour que la similitude reste valable, que les ondes soient du même type, c'est-à-dire de gravité sur le modèle comme dans la nature. Nous allons essayer de dégager les principes qui nous ont conduit à préciser cette échelle.

1. Etude sommaire des ondes soumises à la fois à la pesanteur et à la tension superficielle.

La célérité de telles ondes est donnée par la formule :

$$
\begin{gathered}
C^{2}=\left(g-\frac{\lambda^{\prime}}{2 \pi}+\frac{\mathrm{A} \pi}{\lambda^{\prime}}\right) \text { th } \frac{2 \pi \mathrm{H}}{\lambda^{\prime}} \\
\text { avec } \mathrm{C}=\left(\lambda^{\prime} / \mathrm{T}\right)
\end{gathered}
$$

où $H$ est la profondeur, $\lambda^{\prime}$ la longueur d'onde, $\mathrm{T}$ la période et $\mathrm{C}$ la célérité.

Ia combinaison de (1) el $(2)$ donne:

$$
\frac{1}{\mathrm{~T}_{2}}=\frac{g}{2 \pi \lambda^{\prime}} \text { th } \frac{2 \pi \mathrm{H}}{\lambda^{\prime}}+\frac{\mathrm{A}}{\rho} \frac{2 \pi}{\lambda^{\prime}} \operatorname{th} \frac{2 \pi \mathrm{H}}{\lambda^{\prime}}
$$

Soit $\lambda$ la longueur d'une onde de même période $\mathrm{T}$ mais soumise uniquement à l'action de la pesanteur. Nous supposerons en outre que $\lambda^{\prime}=\lambda+\Delta \lambda$ avec $\Delta \lambda / \lambda$ petit de l'ordre de quelques pour cent. On a dans ces conditions :

$$
\begin{gathered}
\frac{1}{\mathrm{~T}_{2}}=\frac{g}{2 \pi \lambda} \text { th } \frac{2 \pi \mathrm{H}}{\lambda} \\
\frac{1}{\mathrm{~T}_{2}}=\frac{g}{2 \pi \lambda^{\prime}} \text { th } \frac{2 \pi \mathrm{H}}{\lambda^{\prime}}+\frac{\mathrm{A}}{\frac{2}{\lambda_{1}^{\prime} 3}} \text { th } \frac{2 \pi \mathrm{H}}{\lambda^{\prime}}(3) \\
\lambda^{\prime}=\lambda\left(1+\frac{\Delta \lambda}{\lambda}\right)
\end{gathered}
$$

En combinant (3), (4) et (5), on obtient :

$$
\begin{gathered}
1=\left(\frac{1}{1+(\Delta \lambda / \lambda)}+\frac{A}{\rho g} \pm \pi^{2} \frac{1}{\lambda^{2}} \frac{1}{1+3(\Delta \lambda / \lambda)}\right. \\
\frac{\operatorname{th}(2 \pi \mathrm{H} / \lambda)-\operatorname{th}(2 \pi \mathrm{H} / \lambda) \Delta \lambda / \lambda}{1-\operatorname{th}(2 \pi \mathrm{H} / \lambda) \operatorname{th}(2 \pi \mathrm{H} / \lambda) \Delta \lambda / \lambda} \frac{1}{\operatorname{th}(2 \pi \mathrm{H} / \lambda)}
\end{gathered}
$$

A partir de cette équation on pourrait, pour un $\Delta \lambda / \lambda$ donné, se fixer $2 \pi H / \lambda$ et en tirer $\lambda$. On en déduirait alors facilement la longueur d'onde par profondeur infinie, puis la période.

2. Cette méthode étant longue, nous avons décomposé le problème en trois parties:

a) $2 \pi \mathrm{H} / \lambda$ est assez petit pour que la célérité ne dépende pas de la période;

b) $2 \pi \mathrm{H} / \lambda$ est assez grand pour que la célérité ne dépende que de la période;

c) $2 \pi \mathrm{H} / \lambda$ a des valeurs comprises entre ces deux extrêmes.

On obtient pour ces trois cas comme limite :

$$
\begin{aligned}
\mathrm{H}_{\mathrm{em}} \mathrm{T}_{\text {kec }}^{2} & =\frac{1,5510^{-3}}{\Delta \lambda / \lambda} \\
\mathrm{T}_{\text {sec }}^{2} & =0,105(\Delta \lambda / \lambda)^{1 / 4} \\
\lambda_{\mathrm{e} \mathrm{a}}^{2} & =\frac{1}{\Delta \lambda / \lambda \quad 4 \pi \mathrm{H} / \lambda \quad 1 / \mathrm{Sh} 4 \pi \mathrm{H} / \lambda+1}
\end{aligned}
$$

Nous avons reporté ces résultals sur un graphique logarithmique (fig. 2) en portant $T_{\text {see }}$ en abscisses et $H_{\mathrm{cm}}$ en ordonnée pour différentes valeurs de $\Delta \lambda / \lambda$. de 1 à $10 \%$. Les courbes limites se composent sensiblement de deux droites raccordées par des portions de courbes pour les valeurs de $2 \pi \mathrm{H} / \lambda$ intermédiaires.

Nous avons tracé aussi les droites limites des ondes par grandes profondeurs et par très petites profondeurs. On obtient ainsi six zones ca- 
ractéristiques dont trois nous intéressent pratiquement :

\section{Ondes de gravité}

$I_{A}$ La célérité ne dépend que de la profondeur; $I_{1}$ La célérité dépend à la fois de la période et de la profondeur;

Ic La célérité ne dépend que de la période.

Les zones II sont soumises à la fois à la pesanteur et à la tension superficielle.

3. Il est bon également de faire intervenir les paramètres définissant lo déferlement, car ce devait se réserver la possibilite de dépasser ers limites et de reproduire des houles de 4 a $10 \mathrm{se}$ condes. A l'échelle du 1/150", la période la plus courte élail égale sur le modèle à 0,327 secondes. On en déduit facilement sur le graphique la cambrure minima à utiliser, soit de l'ordre de $9 \%$. Pour cette cambrure, la houle est très instable et pratiquement impossible à reproduire sur un modèle à cause des filtres.

Nous avons adopté en définitive le $1 / 100^{\circ}$. La cambrure limite est alors égale à $3 \%$, ce qui est très acceptable.

Pour un modèle à cette échelle el de dimensions relativement réduites, l'amortissement des ondes d'une extrémité à l'aulre est insensible.

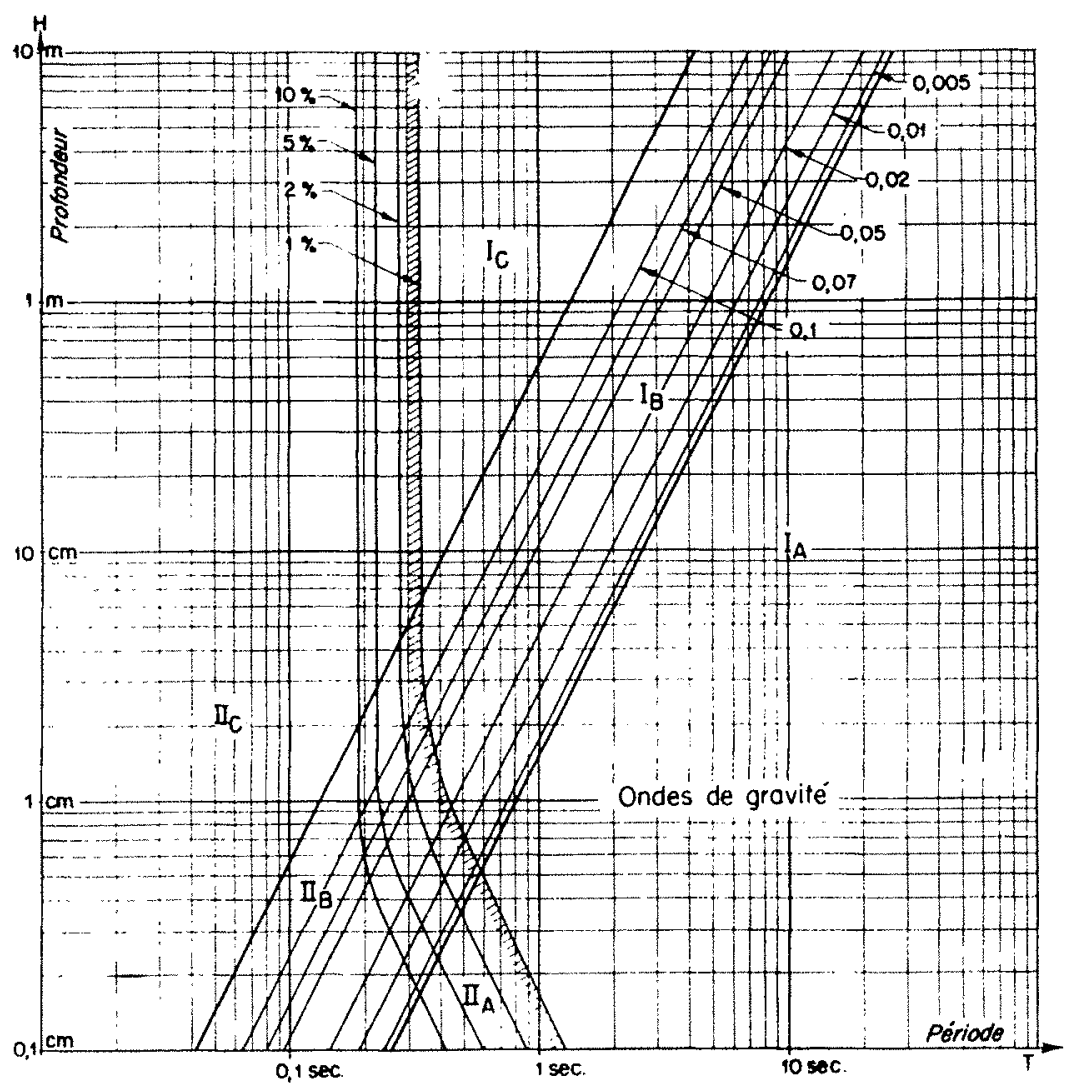

Fig. 2.

Ondes de gravité et

ondes de tension superficielle. phénomène doit se produire avant que les ondes aient pris leur caractère d'ondes de tension superficielle. On sait que la houle brise lorsqu'elle a une cambrure égale à 0,14 th $(2 \pi \mathrm{H} / \lambda)$. On en déduit facilement le rapport $H / \lambda_{y}$ de la profondeur au lieu de déferlement à la longueur d'onde par profondeur infinie pour nne houle de cambrure donnée $\gamma_{0}$ ou large. A $\gamma_{0}$ correspond alu diferlement $\left(\mathrm{H} / \lambda_{0}\right)=k$ d'où $\mathrm{H}=k^{\prime} \mathrm{T}^{2}$. On a ainsi sur le graphique une série de droites tracées pour des valeurs de yo de 0,005 à 0,1 .

4. Application au modèle de Port-en-Bessin Nous avons vu précédemment que les périodes observées allaient de 5 à 9 secondes, mais on

\section{B. - Chorx DEs IIMIJts.}

Les limites du modèle côté large ont été déturminées à partir de plan de vagues qui nous ont permis en outre de siluer les emplacements des générateurs de houle et la largeur des fronts d'onde a reproduire. Devant les volets de ces appareils, des amortisseurs filtrent la houle et suppriment l'influence des réflections sur les ouvrages du port et sur les volets.

\section{C. - REAlisation DU MOdiere.}

Le plan ci-contre (fig. 3) montre les limites 
adoptées en définitive. Deux générateurs de houle permettent de reproduire une largeur de crète de (i $\mathrm{m}$ (600 $\mathrm{m}$ dans la nalure).

L'appareillage de mesures esl de deux sortes:

1. Le «ciel éloilé » qui permel de dégrossir très rapidement les différentes solutions étudiées,

2. Des apparcils de mesure en des points déterminćs : pointes fixes et pointes vibrantes pour les petites amplitudes.

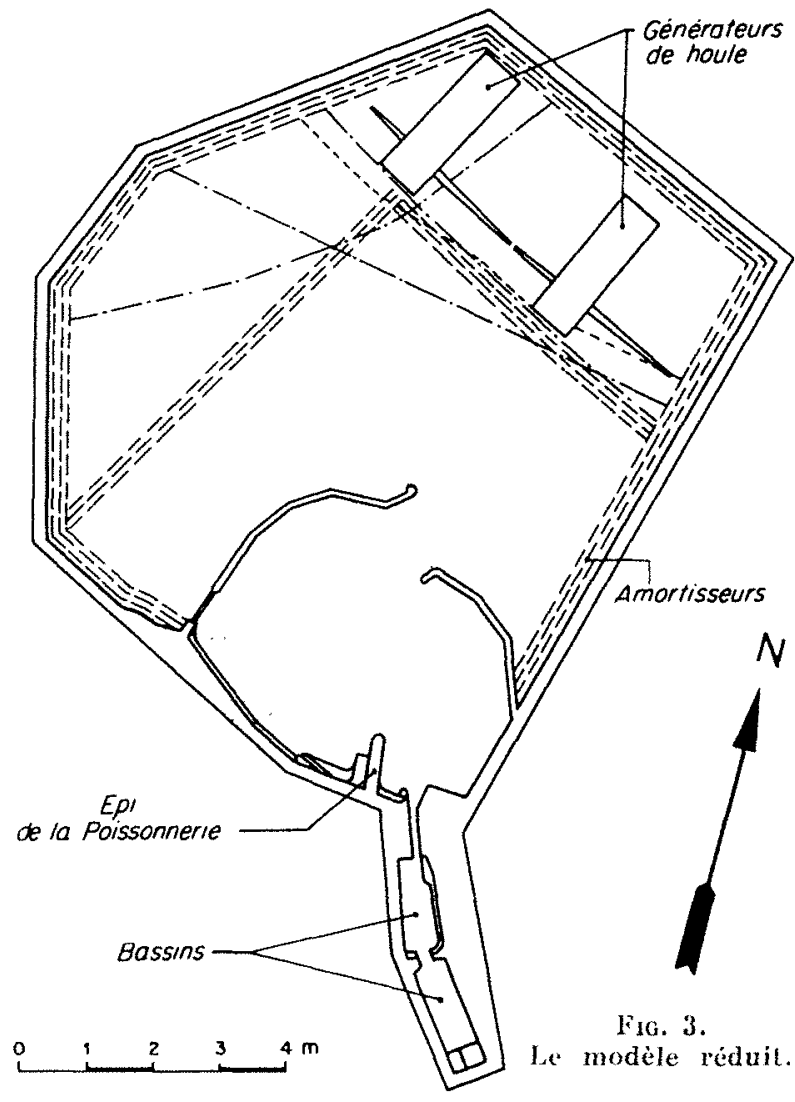

D. - EsSAIS pRÉliminaiREs.

1. Avant de rechercher des solutions pour antéliorer la tenue du plan d'eau, il est nécessaire d'examiner en détail les phénomènes yui se produisent dans le port dans son état actuel. Cette étude a ćté faite sur le modèle mais en liaison étroite avec l'Administration des Ponts et Chaussées pour en comparer les résultats avec les observations naturelles.

Afin de caractériser l'agitalion, les mesures ont été faites en un certain nombre de points typiques.

Les essais ont été effectués en faisant varier systématiquement la période de la houle de 5 à 9 secondes et en déterminant le rapport de l'amplitude de l'agitation en un point déterminé à celle du large.
2. La forme sensiblement circulaire de l'avantport et rectangulaire des bassins, les quais verlicaux favorisent les phénomines de résonance.

Pratiquement, on observe :

a) Un maximum d'agilation tres important atttour de la période de 8 secondes entre l'épi de la Poissonnerie et l'entrée du goulet,

b) Dans le chenal, une légère pointe d'agitation pour 6 secondes,

c) La pénétration de la houle est facilitée par la forme en convergent de l'entrée du chenal.

\section{III. - Les essais de solutions classiques}

\section{A.}

Il résulte de ces observations plusieur's procédés de principe pour diminuer l'agitation :

1. - La modification du tracé des jetées étant difficile et en tout cas trop onéreuse, nous avons cherché à diminuer les réflexions en disposanl des enrochements sur tout ou partie des parois pour absorber l'énergie de la houle incidente.

2. - Pour empêcher l'énergie de se concentrer à l'entrée du goulet, on a songé à créer un bassin protégé à l'intérieur du port. On l'a réalisé, soit en prolongeant en direction de l’épi le môle de la jetée Est, soit en prolongeant dans différentes directions l'épi, soit en construisant une jetée issue d'un point de la jetée Est vers l'épi.

3. - On peut envisager aussi de modifier la passe d'entrée soit en réduisant sa largeur, soit en disposant une entrée en chicane.

4. On peut enfin, pour diminuer l'agitation des bassins seuls, supprimer la forme en conversent de l'entrée du chenal.

\section{B. - LEs RÉsul'tats obtenus :}

Afin de comparer chaque solution, on détermine pour toutes les périodes de seconde en seconde entre 5 et 9 secondes, le coefficient d'amortissement défini par

$$
a_{n}=\frac{\text { amplitude au point } n \text { pour la solution étudice }}{\text { amplitude au point } n \text { pour le port dans son état actuel }}
$$

Pour ces essais comparatifs, l'amplitude de la houle au large est naturellement la inême.

Un grand nombre de solutions ont été essayces; certaines ont été éliminées par l'Administration pour des raisons de navigation et 
nous n'avons retenu en définitive que lrois solutions : $E_{100}$ (prolongation de $100 \mathrm{~m}$ et à 45 degrés de l'épi de la Poissonnerie), $E_{1 \omega} A_{m} /$ (mème prolongation mais complétée par des enrochements disposés le long du quai à profil courbe), et $\mathrm{E}_{100} \mathrm{~A}_{m}$ (même prolongation mais avec des enrochements le long de la totalite des digues). Pour ces trois solutions nous avons les résultats suivants :

Au point 1, point situé à l'extrémité de l'épi de la Poissonnerie :

\begin{tabular}{|c|c|c|c|c|c|}
\hline Périodes $/ \mathrm{s}$ & 5 & 6 & 7 & 8 & 9 \\
\hline $\mathrm{E}_{100} \ldots \ldots \ldots$ & 0,5 & 0,76 & 0,7 & 1,05 & 0,74 \\
$\mathrm{E}_{100} \mathrm{~A}_{m} f \ldots \ldots$ & 0,1 & 0,5 & 0,5 & 0,65 & 0,45 \\
$\mathrm{E}_{100} \mathrm{~A}_{m} \ldots \ldots$ & 0,15 & 0,47 & 0,5 & 0,6 & 0,55 \\
\hline
\end{tabular}

Au point 2, situé dans le premier bassin :

\begin{tabular}{|c|c|c|c|c|c|}
\hline Periodes $/ \mathrm{s}$ & 5 & 6 & 7 & 8 & 9 \\
\hline $\mathrm{E}_{100} \ldots \ldots \ldots$ & 0 & 0,31 & 0 & 0,55 & 0,5 \\
$\mathrm{E}_{100} \mathrm{~A}_{m} f \ldots$ & 0 & 0,05 & 0 & 0,1 & 0,07 \\
$\mathrm{E}_{100} \mathrm{~A}_{m} \ldots \ldots$ & 0 & 0,02 & 0 & 0,07 & 0,04 \\
\hline
\end{tabular}

Pour les bassins seuls, notons que la suppression du convergent donne des valeurs de $a_{n}$ comprises entre 0,5 et 0,6 . Mais, dans lavant-port, $a_{n}$ est de l'ordie de 0,9 a 1,1 , c'est-à-dire que dans certains cas l'agitation est alors un peu auginentéc.

On remarque que dans loutes ees solutions, qui consistent à faire un avant-port partiel dans l'avant-port actuel, les bassins sont tris bien protégés, mais à l'exlérieur on a encore úne agitation assez importante particulierement pour 8 secondes, la résonance n’a pas été supprimée.

Nous avons alors cherché à parfaire cette solution par des ouvrages d'un type nouveau mis au point au Laboratoire et appelés « résonateurs".

\section{IV. - Principe de fonctionnement des résonateurs}

On peut montrer que si des ondes de gravité se propagent dans un canal de faible profondeur, on a les relations suivantes entre la surélévation et la vitesse des particules.

$$
\begin{aligned}
u & =\mathrm{F}[t-(x / c)]+f(l+(x / c)] \\
g / c h & =\mathrm{F}[t-(x / c)]-f[t+(x / c)]
\end{aligned}
$$

on peut généraliser cette formule au cas donde de gravité par profondeur quelconque en rapportant tous les calculs à une vitesse de référence quelconque, par exemple la vitesse moyenne ou la vitesse de surface. Avec la vilesse de surface on obtient ainsi :

$$
\begin{aligned}
h \frac{2 \pi}{\mathrm{T}} \operatorname{lh} \frac{2 \pi \mathrm{H}}{\lambda} & =\mathrm{F}[t-(x / c)]-f[t+(x / c)] \\
u & =\mathrm{F}[t-(x / c)]+f[t+(x / c)]
\end{aligned}
$$

Dans tous les cas on peut done ecrire:

$$
\left\{\begin{aligned}
k h & =\mathrm{F}[t-(x / c)]-f[t+(x / c)] \\
u & =\mathrm{F}[t-(x / c)]+f[t+(x / c)]
\end{aligned}\right.
$$

Considérons maintenant (fig. 4) dans un canal un obstacle d'abscisse L. Soit $h$ et $\|$ les valeurs

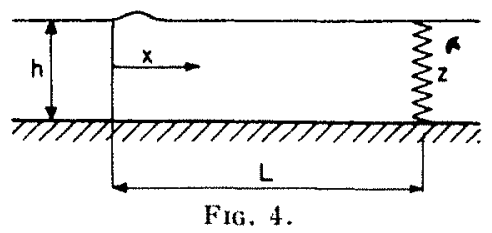

de la surélívation et de la vitesse dans cette seclion. Les equations ci-dessus deviennent :

$$
\begin{aligned}
k h & =\mathrm{F}[(t-\mathrm{L} / \mathrm{c})]-f[(t+\mathrm{L} / \mathrm{c})] \\
u & =\mathrm{F}[(t-\mathrm{L} / c)]+f[(t+\mathrm{L} / \mathrm{c})]
\end{aligned}
$$

que nous écrivons pour simplifier :

$$
\begin{aligned}
k h & =\mathrm{F} \cdots-f \\
l & =\mathrm{F}+1
\end{aligned}
$$

Posons $(h / u)=z$ que nots appellerons impédance par analogie avec l'électricité $z$ est en général une fonction complexe de $h, \mathrm{H}, \mathrm{T}$, mais nous verrons plus loin que, dans certains cas, elle a une forme lout a fait analogue à une impédance électrique :

$$
Z=r+j[\mathrm{~L},(\omega) \cdots(1 / c(\omega))]
$$

Si $h=u z$, on peut en deduire:

$$
\begin{aligned}
& \quad l=\mathrm{F}(1-k z) /(1+k z)=\mathrm{F}(1 \cdots x) /(1+x) \\
& \text { avec }: x=k z \text {. }
\end{aligned}
$$

Certains cas particuliers sont intéressants : $z \nsim$ (cas d'un mur vertical), $f=-\mathrm{F} ; z=0$ (canal débouchant dans un grand réservoir) $f=F$; cas de l'impérance itérative $x=1 \quad f=0$. 


\section{2. .-. Singularité disposée latéralement (fig. 5).}

Supposons un canal infini on fermé sur son impédance itérative sur le còté duquel est dis-

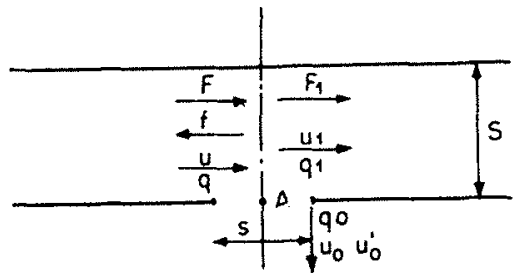

Fic. 5.

posée une singularité. D'après la notation de la figure, on peut écrire, $q$ étant le débit :

$$
q=u \mathrm{~S}, \quad q_{1}=u_{1} \mathrm{~S}, \quad q_{0}=u_{0}^{\prime} \mathrm{S} .
$$

avec :

$$
u_{0}=u_{0}^{\prime}(s / \mathrm{S})=\left(u_{0}^{\prime} / \sigma\right)
$$

On peut aussi écrire :

avec :

$$
q_{0}=u_{1} \mathrm{~S}
$$$$
\sigma=\mathrm{S} / \mathrm{s} \text {. }
$$

On a, entre les différentes ondes, les relations suivantes :

$g(h / c)=\mathrm{F}-f=\mathrm{F}_{1} \quad u=u_{1}+u_{0} \quad u_{0}=(h / z)$

On en tire :

$$
\mathrm{F}_{1}=\mathrm{F}_{2 x} /(1+2 x) \quad f=\mathrm{F}_{1 /(1+2 \alpha)}
$$

Ainsi, pour protéger l'aval de $A, \alpha$ devra ètro faible. Nous avons dit que, dans certains cas, on peul écrire $: z \cong r+j[\mathrm{~L} \omega-(1 / c \omega)]$. Pour que $\mathrm{F}_{1}$ soit faible, il faudra que $r$ soit petil et que $\mathrm{I}=2 \pi \sqrt{\mathrm{L} c}$. Lorsque la période de la houle est égale ou voisine à la période de résonance du système, l'onde restante $F_{1}$ est très faible. D'où le nom de « résonateurs » donné à ces appareils.

\section{3. -- Résonateurs en charge.}

M. Valembois, qui a eu l'idée de ces appareils et du principe de leur fonctionnement, a proposé d'utiliser conme résonateurs des puits oscillants.

L'équation du mouvement dans ces appareils est de la forme :

$$
\mathrm{M}\left(d^{2} z / d t^{2}\right)+f\left(d^{2} / d t\right)^{2}+k z=k^{\prime} h
$$

Pour les petites oscillalions, on peut admettre que le terme de perte de charge est linéaire. On obtient alors une équation de même forme que celle d'un circuit électrique comprenant résistance, self, capacité.

La période propre d'un tel système est égale à $\mathrm{T}_{n}=2 \pi \sqrt{I / g}$ où $l$ représente la longueur de la colonne liquide oscillante. L'efficacité est proportionnelle à la section d'entrée. Nous avons essayé cet appareil. II donne de bons résultats mais i) est assez difficile de faire varier ' $T_{31}$.

\section{4. - Résonateurs ḋ surface libre.}

$A u$ cours d'essais, nous avons remarqué qu'un bassin rectangulaire disposé latéralement à un canal produisait pour certaines périodes une diminution de l'amplitude de la houle à l'aval. Pour ces périodes, le bassin entre en résonance et la résonance en quart d'onde est particulièrement intéressante.

L'étude du mouvement pour ce système montre que l'on a, en faisant la même hypothèse de pertes de charge linéaires, des équations tout à fait analogues. Mais le gros intérêt de ce type de résonateur est de pouvoir régler très facilement sa période propre puisqu'il suffit de fixer la dimension transversale du résonateur à un quart de longueur d'onde.

\section{5. -_ Combinaisons de résonateurs.}

Lorsqu'un résonateur est placé sur un canal fermé, non plus par son impédance itérative, mais par une paroi verticale, le résonateur doit être placé à une distance de paroi égale à $k(\lambda / 2)$ ou $k=0,1,2 \ldots$ Il faut, en outre, que $k$ ait la valeur la plus faible possible compatible avec l'ensemble de l'installation.

Lorsqu'on veut protéger un ouvrage pour unc samme étendue de période, on dispose un certain nombre de résonateurs dont on peut déterminer par le calcul les positions les plus efficaces, mais la complication croît très rapidement. On ne peut alors rechercher la solution optima que par expérience sur modèle réduit.

\section{Application à la protection de l'avant port}

1. - Avant-port.

a) Nous avons d'abord cherchè à compléter la solution du type $\mathrm{E}_{100} \mathrm{~A}_{m}$ par deux résonateurs face à face accordés pour la période de 7 secondes, c'est-à-dire par une période dangereuse et très voisine de la période de résonance de 8 secondes. Les résultats obtenus sont très nets.

\begin{tabular}{|c|c|c|c|c|c|}
\hline Periodes $/ \mathrm{s}$ & 5 & 6 & 7 & 8 & 9 \\
$\ldots$ & & & & & \\
$\mathrm{E}_{100} \mathrm{R} \mathrm{A} \mathrm{A}_{m} f \ldots$ & 0 & 0,25 & 0,33 & 0,4 & 0,36 \\
$\mathrm{E}_{100} \mathrm{~A}_{m} f \ldots$ & 0,1 & 0,5 & 0,5 & 0,65 & 0,45 \\
$\mathrm{E}_{1 \ldots 10} \mathrm{R} \mathrm{A}_{m} \ldots$ & 0,15 & 0,47 & 0,5 & 0,6 & 0,55 \\
\hline
\end{tabular}


On constate la nette amélioration obtenue avec ce dispositif même pour l'ensemble des périodes, car les résonateurs agissent non seulement pour la période d'accord, mais aussi pour les périodes voisines.

b) En ajoutanl aux deux résonateurs accordès pour 7 secondes, deux autres accordés l'un sur 8 secondes et l'autre sur 9 secondes, on obtient :

\begin{tabular}{|c|c:c:c:cc|}
\hline Périodes $/ \mathrm{s}$ & 5 & 0 & 7 & $s$ & 9 \\
& & & & & \\
\hline $\mathrm{E}_{100}$ D R A & 0 & 0,07 & 0.25 & 0,39 & 0,36 \\
$\mathrm{E}_{100}$ D R A & 0,28 & 0,03 & 0,23 & 0,43 & 0,02 \\
\hline
\end{tabular}

Ces deux solutions sont done très satisfaisantes.

a) Les deux tableaux ci-dessous montrent l'influence des résonateurs sur les bassins pour les deux solutions $A_{m} f$ et $A_{m}$. On arrive pratiquement a une agitation si faible qu'elle n'est plus mesurable.

\begin{tabular}{|ccccc|ccc|}
\hline Périodes/s & 5 & 6 & 7 & 8 & 9 \\
$\mathrm{E}_{1,10} \mathrm{~A}_{m} / \ldots$ & 0 & 0 & 0 & 0,1 & 0,07 \\
$\mathrm{E}_{100} \mathrm{R} \mathrm{A}_{m} / \ldots$ & 0 & 0 & 0 & 0,03 & 0,03 \\
$\mathrm{E}_{100} \mathrm{D} \mathrm{R} \mathrm{A} \mathrm{A}_{m} /$ & 0 & 0 & 0.01 & 0,03 & 0,01 \\
\hline
\end{tabular}

\begin{tabular}{|c|c|c|c|c|c|}
\hline Périodes/s & 5 & 6 & 7 & $s$ & 9 \\
\hline $\mathrm{E}_{1 \oplus 0} \mathrm{~A}_{m} \ldots$ & 0 & 0,02 & 0 & 0,07 & 0,04 \\
\hline $\mathrm{E}_{10 \mathrm{n}} \mathrm{R} \mathrm{A}_{m} \ldots$ & 0 & (1) & 0 & 0 & 0 \\
\hline$E_{1 m}$ D R A $A_{m !}$ & 0 & 0 & 0 & 0 & 1) \\
\hline
\end{tabular}

b) Nous avons enfin étudié (lig. (i) une solulion partielle destince uniquement it diminner l'agitation dans les bassins. Elle est constituée par la suppression du convergent à l'entrée du chenal et par deux résonateurs accordés sur 7

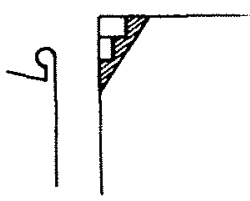

FIG. 6.

et 8 secondes, disposés d'un même côté. Celte solution peut ètre complétée éventuellement par les talus amortisseurs de l'avant-port.

On obtient ainsi :

\begin{tabular}{|c|c|c|c|c|c|c|c|}
\hline Périodes/s & 5 & $\vdots$ & i & 7 & & $s$ & 9 \\
\hline RC...... & 0 & & 0 & 0 & 1 & 0,1 & 0,2 \\
\hline $\mathrm{I} \mathrm{CAA}_{m} / \ldots$ & 0 & & 11 & 0 & 1 & 0 & 0 \\
\hline $\mathrm{RC} \mathrm{A}_{m} \ldots \ldots$ & 0 & 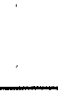 & 0 & 0 & 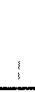 & 0 & 0 \\
\hline
\end{tabular}

Le seul reproche que l'on puisse faire à ce dispositif est le clapotis qui se forme devant le chenal et qui risque de gêner l'entrée des bateaux. Une estacade en pieux faciliterait vraisemblablement la pénétration des navires sans nuire a l'amélioration obtenue.

\section{VI. - Conclusion}

Ainsi les résonateurs permettent, en réfléchissant. la houle incidente, de protéger efficacement les ouvrages d'un porl. De construction très simple et peu onéreux, ils peuvent se combiner facilement avec d'autres ouvrages. Leur emploi n'est limité que par la largeur du chenal à proléger qui ne doit pas etre trop srande par rapport à la longueur d'onde de la houle.

\section{DISCUSSION}

M. le Président HupNen remercie M. Bmand de sa très intéressante communication. Il rappelle que le problème pratique du port de Port-en-Bessin avait été posé par M. Jouveneaux, Ingénieur en Chef des Ponts et Chaussées du Calvados.

Sur sa demande, M. Binard précise que les donnces de l'étude en Laboratoire, communiquées par l'Administration des Ponts et Chaussées à la suite d'une campagne de mesures, faisaient ressortir:
11) Devant l'entree, l'existence do houles de $3 \mathrm{~m}$ d'ampli tude avec creux encore plus important a plein. mer;

b) A l'avant-port, en particulier pour la période de 8 secondes, des creux de l'ordre de $2 \mathrm{~m}$,

c) Dans les bassins: des creux de l'order de $0,70 \mathrm{~m}$ pour le premier et do $0,40 \mathrm{~m}$ a $0,50 \mathrm{~m}$ pour le second. 
M. Bmano ajoute que, bien que très atlenuée, l'agllation dans les bassins est encore assez dangereuse par suite de l'entassement des bateaux.

Les instructions nautiques recommandent de ne pas relâcher dans ee port. Cependant, l'agitation n'est dangereuse qu'a partir de la cote +7 par suite de fonds issez élevés et c'est ce qui explique que tous les essais en Laboratoire ont eté faits à pleine mer.

M. Brrard confirme que les amortisseurs hydrauliques sont du même type que ceux utilisés par l'étude du port de Dunkerque et presentés an Laboratoire de Chatou lors de la visite du 20 novembre 1953 , et que le ciel étoilé a été utilisé pour une houle de période égale a 9 secondes, et de direction Nord-Est, qui est la plus défavorable.

En réponse à une question de M. Devimeux, M. Bhand arécise que l'efficacité des résonateurs décroit rapidement lorsque la largeur de la passe d'entréc devient supérieure à la longueur d'onde de la houle incidente. Dans le cas particulier de Port-en-Bessin, la passe d'enrée a une largeur de $100 \mathrm{~m}$ sensiblement égale à la longueur d'onde de la houle incidente, de période 9 secondes et l'expérience a montré que, effectivement, l'efficacité des résonateurs diminuait rapidement si l'on élargissait la passe d'entrée.

M. Devineux signale à ce propos que sur le modèle du port de Monaco, essayé à Maisons-Alfort, les résultats n'ont pas été intéressants pour une largeur de $130 \mathrm{~m}$ et une houle assez courte.

M. Devmevx attire l'attention de M. Brand sur le fait que les ouvragses résonnants réduisent, par leur présence, la largenr de la passe d'entrée et que l'agitation dans le port doit s'en trouver réduite, abstraction faite de tout effet de résonance proprement dit. Aussi il demande à Mr. Brand si les expériences ont permis de distinguer les réductions d'agitation dues à chacun de ces deux (ffets et de mettre en évidence le hénéfle qui résulte de l'emploi des résonateurs.

Répondant à cette deuxième question de M. Devimeux, M. Biraro précise que la comparaison des mesures de l'agitation, faites d'une part en l'absence des résonateurs, d'autre part avec ces appareils, et, par ailleurs, avec les mêmes dispositions d'ouvrages dans les deux cas, permet d'attribuer aux résonateurs une réduction de l'amplitude au tiers, environ.

\section{QUATRIEMES JOURNÉES DE L'HYDRAULLQUE Paris -- Été 1956}

La Société Hydrotechnique de France organise les

QUATRIÈMES JOURNÉES DE L'HYDRAULIQUE,

qui auront lieu au cours de l'été I956.

Le sujet étudié au cours de ces Journées sera:

\section{LES ÉNERGIES DE LA MER :}

\section{Houle, Marée, Energie thermique}

Un programme précisant les questions mises à l'étude sera diffusé prochainement.

Les séances de travail auront lieu à Paris et seront associées à un voyage d'étude approprié au sujet.

Les dates exactes des IV Journéeś de L'Hydraulique et le but du voyage d'étude seront fixés ultérieurement.

Les personnes désireuses de présenter des mémoires ou de prendre part aux discussions des IVo Journérs de L'HYdraulique sont priées de le faire connaître dès maintenant à la Société Hydrotechnique de France.

Un résumé en dix lignes de chaque mémoire devra être adressé en trois exemplaires à la Société Hydrotechnique de France, avant le $I^{\text {er }}$ janvier 1956. Les mémoires retenus devront être remis in extenso en dix exemplaires trois mois au plus tard avant les " Journées". 